\title{
Lenguaje y comunicación componentes importantes para el desarrollo del bienestar infantil
}

\author{
Language and communication important components for the development \\ of child well-being \\ Linguagem e comunicação Componentes importantes para o desenvolvimento \\ do bem-estar infantil
}

\section{ARTICULO DE INVESTIGACIÓN}

\section{Diana Carolina Ortiz Delgado}

dortizd@unemi.edu.ec

ORCID: 0000-0003-4011-6334

Maryuri Edid Cortez Moran mcortezm@unemi.edu.ec

ORCID: 0000-0003-2306-7331
Erika Marissa Ruperti Lucero

erupertil@unemi.edu.ec

ORCID: 0000-0002-1376-0143

\author{
Abraham Clemente Varas Santafé \\ abraham.varas@estudiante.uam.es \\ ORCID: 0000-0002-1961-7524
}

\section{Universidad Estatal de Milagro, Ecuador}

Recibido 07 de noviembre 2020 | Arbitrado y aceptado 26 de noviembre 2020 | Publicado en diciembre 2020

\section{RESUMEN}

El bienestar infantil conlleva algunas facetas que se integran para brindar al infante las herramientas para su desarrollo óptimo. El objetivo del estudio es describir las características lingüísticas en niños y niñas entre 4 a 6 años de edad para identificar los predictores del óptimo desarrollo del lenguaje como componente importante del bienestar infantil. La muestra está conformada por 200 participantes escolarizados en centros educativos. Entre los principales resultados se identificó que los participantes que han recibido espacios de estimulación temprana presentan mejores niveles de madurez en el lenguaje. Un predictor importante para la comunicación es el nivel madurativo del lenguaje articulado. Una de las limitaciones del estudio fue no contar con el mismo número de participantes por grupo de edad, pero el presente estudio brinda lineamientos importantes para profundizar sobre la temática y activar líneas de acción en el bienestar infantil y la estimulación en el lenguaje a edades tempranas.

Palabras clave: Desarrollo; lenguaje; bienestar, infancia

\section{ABSTRACT}

Child welfare involves some facets that are integrated to provide the infant with the tools for optimal development. The objective of the study is to describe the linguistic characteristics in boys and girls between 4 and 6 years of age to identify the predictors of optimal language development as an important component of child well-being. The sample is made up of 200 participants enrolled in educational centers. Among the main results, it was identified that the participants who have received early stimulation spaces present better levels of maturity in language. An important predictor for communication is the maturational level of articulate language. One of the limitations of the study was not having the same number of participants by age group, but the present study provides important guidelines to deepen on the subject and activate lines of action in child well-being and stimulation in language at an early age.

Key words: Development, language, well-being, childhood 


\section{RESUMO}

O bem-estar infantil envolve algumas facetas integradas para fornecer à criança as ferramentas para um desenvolvimento ideal. 0 objetivo do estudo é descrever as características linguísticas em meninos e meninas entre 4 e 6 anos de idade para identificar os preditores do desenvolvimento ideal da linguagem como um componente importante do bem-estar infantil. A amostra é composta por 200 participantes matriculados em centros de ensino. Dentre os principais resultados, identificou-se que os participantes que receberam espaços de estimulação precoce apresentam melhores níveis de maturidade na linguagem. Um preditor importante para a comunicação é o nível de maturação da linguagem articulada. Uma das limitações do estudo foi não ter o mesmo número de participantes por faixa etária, mas o presente estudo fornece orientações importantes para se aprofundar no assunto e ativar linhas de ação no bem-estar infantil e estimulação da linguagem desde a infância.

Palavras-chave: Desenvolvimento; língua; bem estar infância

\section{INTRODUCCIÓN}

El infante posee un sin número de etapas y procesos por los que debe trascurrir de forma efectiva para su desarrollo, en cada una de estas, es imprescindible que se promocione el bienestar de los mismos en todo aspecto, tanto físico, psicológico y emocional. El bienestar de forma general puede relacionárselo con las condiciones positivas en la que se encuentra el sujeto tanto en vivienda, educación, salud, emoción, entre otras, es decir, el término bienestar no solo está sujeto a lo material, sino también a lo impalpable pero imprescindible para la vida del ser humano (Castilla, 2014).

El desarrollo o traspaso del infante por varios procesos, hace parte de su naturalidad humana, de esto depende que el individuo pueda encajar en una sociedad, desenvolverse y ser aceptado por la misma, ya que de ella se aprende el lenguaje, cultura e incluso, la conocida identidad cultural, que permite identificar a ciertas personas dentro de una comunidad otorgándole posteriormente la necesidad de obtener un auto concepto basándose en lo aprendido dentro de la misma sociedad.

En base a ello el presente estudio tiene como objetivo describir las características lingüísticas adquiridas en niños y niñas entre 4 a 6 años de edad para identificar los predictores del óptimo desarrollo del lenguaje como componente importante del bienestar infantil. Entre estas características que se busca exponer se evidencian el bienestar infantil, el lenguaje y los factores intrínsecos y extrínsecos que tienen que ver con su desarrollo en todos los aspectos.

Es imprescindible que se estudie de forma más detallada el proceso del lenguaje y como su adquisición influye en el bienestar infantil, debido a que, al ser un proceso normal $\mathrm{y}$ natural, genera mucha expectativa, aunque cuando se da no se produzca asombro. Sin embargo, es necesario que todo ser humano desarrolle este aspecto, su retraso puede causar dificultades para el infante que puede afectar su bienestar, inclusive no solo su retraso sino también su ausencia y sobre todo esta, provoca severos problemas en el desenvolvimiento del sujeto dentro de la sociedad general.

En bienestar infantil se encuentran inmersos componentes importantes como lo son la familia, el desarrollo del mismo, el juego, la escuela, compañeros, amigos o sociedad en general que son quienes formarán parte de su aprendizaje y consolidación de la personalidad, carácter e identidad. En el desarrollo se evidencia marcada la adquisición del lenguaje como proceso natural y normal de todo ser humano (Chávez, et al., 2012).

El lenguaje en el ser humano aparece a partir del primer año de vida, su adquisición aparentemente se da sin esfuerzo ya que todo individuo lo aprende con su entorno. Sin embargo, su aparición no conlleva una atención excesiva, a pesar de ello esta etapa da seguridad 
en cuanto al desarrollo del sujeto y alegría al entorno que rodea al infante. Sin embargo, la comprensión completa de este proceso si conlleva una importante responsabilidad tanto para el menor como para el adulto que busca que este aprendizaje se dé sin errores, por lo que se considera un trabajo conjunto y no únicamente del niño (Díez, Pacheco, De Caso, García, y García, 2009). El lenguaje está íntimamente relacionado con las disciplinas académicas, por lo cual ayuda a la construcción del aprendizaje y con ello a la adquisición del conocimiento así como la puesta en práctica del mismo (Espinoza, Marco, y Ygual, 2017).

Este proceso se debe desarrollar de forma normal en todos los seres humanos debido a que se evidencia su aparición gracias al aprendizaje vicario o por imitación, de tal forma que su aparición es de forma normal. El ser humano, por naturaleza, necesita comunicarse con su entorno y su medio. La comunicación ha sido parte de la historia humana y ha sido parte importante del desarrollo y progreso de las sociedades y comunidades. El lenguaje se presenta de diferentes formas, tales como, la comunicación no verbal, verbal que puede ser expresiva y receptiva, expresión emocional y el interés y exploración, las cuales permiten al ser humano comunicarse de maneras diversas (Vargas, et al., 2016).

El lenguaje oral, es una de las herramientas más imprescindible para la comunicación, por lo que juega un papel muy importante para el aprendizaje y mucho más en la etapa escolar, pero también es significativo para el futuro tanto personal como profesional de todo ser humano. Como ya se ha explicado con anterioridad, la adquisición de este en el caso de los niños, se da de forma natural, generalmente los niños adquieren un domino de su propia lengua con el uso del medio lingüístico que lo rodea. Científicamente hablando, el desarrollo del lenguaje oral y a su vez del habla se da a través de las diferentes competencias cognitivas que forman parte de las funciones ejecutivas, en relación con las redes neuronales que captan el estímulo indicado para la producción de este en base a la necesidad de comunicación entre sus familiares y sociedad en general (Billard, 2014).

Campo (2009), expresa que en de forma general, los niños y niñas que se encuentran dentro de las edades de entre 3 y 7 años, se hallan en la conocida etapa de niñez temprana, la cual está caracterizada por procesos de gran importancia en el desarrollo de su pensamiento, memoria y por supuesto lenguaje. A esta edad se puede evidenciar una mayor capacidad para procesar todo tipo de información en base a las conexiones cerebrales y sus componentes neurobiológicos.

En el proceso del lenguaje se pueden ver actuar una serie de elementos neurofisiológicos que le permiten al sujeto decodificar y producir el mismo. Entre los que se pueden notar, son el sentido de la audición que le permite al individuo poder escuchar lo que le dice o está en su medio, el cerebro que es quien decodifica a través de la corteza cerebral la información captada por los sentidos, que a su vez originan señales que son enviadas a los órganos fonatorios para la producción del lenguaje (Vives, 2002).

En el proceso de la adquisición del lenguaje, también influye mucho las Funciones ejecutivas (FE), las cuales pueden ser conceptualizadas según, como un conjunto de procesos cognitivos, afectivos y motivacionales que se encuentran expresamente destinadas a controlar el consiente de todo sujeto y por ende su pensamiento, gran parte de la comunicación también depende del desarrollo de las FE. Estas pueden darse durante mas formalmente a partir del primer año de vida, esto se conoce debido a que diversos estudios han demostrado que durante esta etapa el infante logra desarrollar actividades ejecutivas como el 
retener ciento tipo de información en períodos de tiempos cortos, lo cual tiene mucha relación con la memoria, que forma parte de los procesos que lleva a cabo la FE (Lepe, Pérez, Rojas, y Ramos, 2018).

De acuerdo con los niños y niñas aprenden el lenguaje con la interacción que mantienen con las personas que forman parte del entorno que los rodea, así como la interacción familiar que posea; específicamente la figura de apego que mantenga es primordial para el refuerzo del uso del lenguaje del infante y la redirección del mismo, en cuanto al uso espontaneo que el niño o niña haga del lenguaje aprendido. De forma que, mediante este refuerzo con su figura de apego, se puede analizar si el uso del lenguaje por parte del niño está o no relacionado con la situación en la que se encuentran, determinando si existe fallas de uso o a su vez un desarrollo normal y correcto del mismo (Moreno, García, Godoy, y Suárez, 2011). El niño pasa por distintas etapas desde los 0 hasta los 36 meses, estas etapas de interacción lingüísticas prelocutivas, transaccionales e intencionales se convierten en base importante de la adquisición del lenguaje materno en sus componentes fonológicos, semánticos y pragmáticos del lenguaje. (Canales, Velarde, Meléndez y Ligán, 2014)

El lenguaje a su vez posee manifestaciones específicas, tales como el articulado, comprensivo y expresivo, que permiten determinados tipos de comunicación, las cuales permiten separar al hombre como seres racionales, tal como el conocido lenguaje articulado que difiere del resto de animales irracionales, siendo uno de los elementos diferenciales principales, el habla, en base a que la lengua humana, aparte de cumplir sus funciones gustativas, permite la emoción de sonidos articulados que permite la diferenciación de otros animales con las que el ser humano si comparte ciertos sentidos como la vista, oído, tacto, entre otros (Montoya, 2003).
El lenguaje comprensivo se presenta desde su inicio a través de 3 formas, la no lingüística que se activa por medio de un mensaje que se quiere dar y que tiene la obligación de estar claro para establecer una interacción con el entorno, la forma lingüística que permite la deducción del mensaje que se desea dar a través de la comprensión de las palabras y su uso en contexto, punto en el cual entra la 3era forma que se refiere al análisis morfosintáctico y léxico (Paredes y Del Rosario, 2014). Por lo que se puede deducir que el lenguaje comprensivo es más bien el proceso en el que el ser humano extrae los significados de los mensajes que recibe durante la comunicación para un análisis de su léxico y contexto que le permita comprender de qué habla el emisor o qué quiso dar a expresar con su mensaje.

En cuanto al lenguaje expresivo, este posee una íntima relación con el comprensivo. Se refiere al proceso de índole neuropsicológico que les permite a los niños comunicarse, adquirir aprendizajes, organizar sus pensamientos y regular su comportamiento, entre otros aspectos importantes de todo ser humano. Dentro de la educación infantil, el lenguaje expresivo oral comienza cuando se evidencia las primeras expresiones del sujeto incluso desde sus primeros balbuceos que suelen darse a edades de entre 2 y 3 años, edad en la que justo están en la adquisición del lenguaje, por lo que el aumento del léxico forma parte de sí mismos (Albets y De la Peña, 2016).

Sin embargo, este proceso de adquisición del lenguaje puede verse seriamente afectado por los contenidos psicopatológicos que se presentan con frecuencia en TV y que no poseen un control por parte de los padres, representantes $\mathrm{o}$ tutores. Este tipo de contenidos, afectan empobreciendo el lenguaje de los niños, el cual se puede reducir a códigos que se encuentran restringidos o la utilización de lenguaje extranjero en una mezcla con el 
propio, perdiendo la identidad lingüística de su cultura y sociedad (Pedreira, 2006).

A pesar de ello, esto puede evitarse con el debido control de lo que ven los niños y niñas, así como a través de la estimulación temprana del lenguaje. El desarrollar espacio lectores, permite también una adquisición del lenguaje favorable, según (Ripalda, Macías, y Sánchez, 2020), expresan que los espacios deben estar adecuados para despertar el interés del infante, de forma que puedan escoger entre géneros literarios, personajes y autores. Condo (2019) comenta que "la lectura es una actividad importante y útil que las personas adquieren en su vida, entendiendo por ésta como un proceso complejo de decodificación e interpretación de signos". (p. 267)

Es decir, es imprescindible principalmente comprender que la estimulación temprana produce un impacto significativo en el crecimiento o desarrollo del bebe y del infante posteriormente, muchas veces, este proceso aporta una preparación al sujeto para enfrentarse a lo que por naturaleza debe hacer como caminar, correr, hablar, expresarse, comunicar, leer, escribir, entre otros sin número de procesos que son parte de la formación de todo ser humano para su desenvolvimiento en interacción con el medio que lo rodea (González Zúñiga Godoy, 2007).

\section{MÉTODO}

La metodología utilizada en el presente trabajo se basó en el enfoque cuantitativo de índole trasversal correlacional a través de la aplicación de instrumentos de medición: Cuestionario de madurez infantil (CUMANIN) dónde las variables fueron medidas por medio de las sub pruebas de Lenguaje Articulado, Lenguaje Expresivo y Lenguaje Comprensivo. Además, se aplicó una encuesta sociodemográfica para obtener datos sobre edad de los niños y niñas, información de antecedentes de asistencia a actividades de estimulación temprana y datos del desarrollo motor. De acuerdo a los principios de ética se extendió la información de la intención de la investigación a los cuidadores legales, posterior a aquello se firmó el consentimiento informado para la participación de los niños y niñas en el estudio. La aplicación de la prueba fue individual, los criterios de inclusión de la muestra fueron: niños y niñas que tengan entre 4 a 6 años de edad, que hayan completado todas las sub pruebas del instrumento. Es así que la muestra se compuso de 200 niños $(46,50 \%$ de niñas y $53,50 \%$ de niños).

\section{RESULTADOS}

En los resultados se puede apreciar que el componente de lenguaje articulado que el $54,30 \%$ de los participantes presentó un nivel de madurez en el lenguaje de acuerdo a su rango de edad (Gráfico 1). 


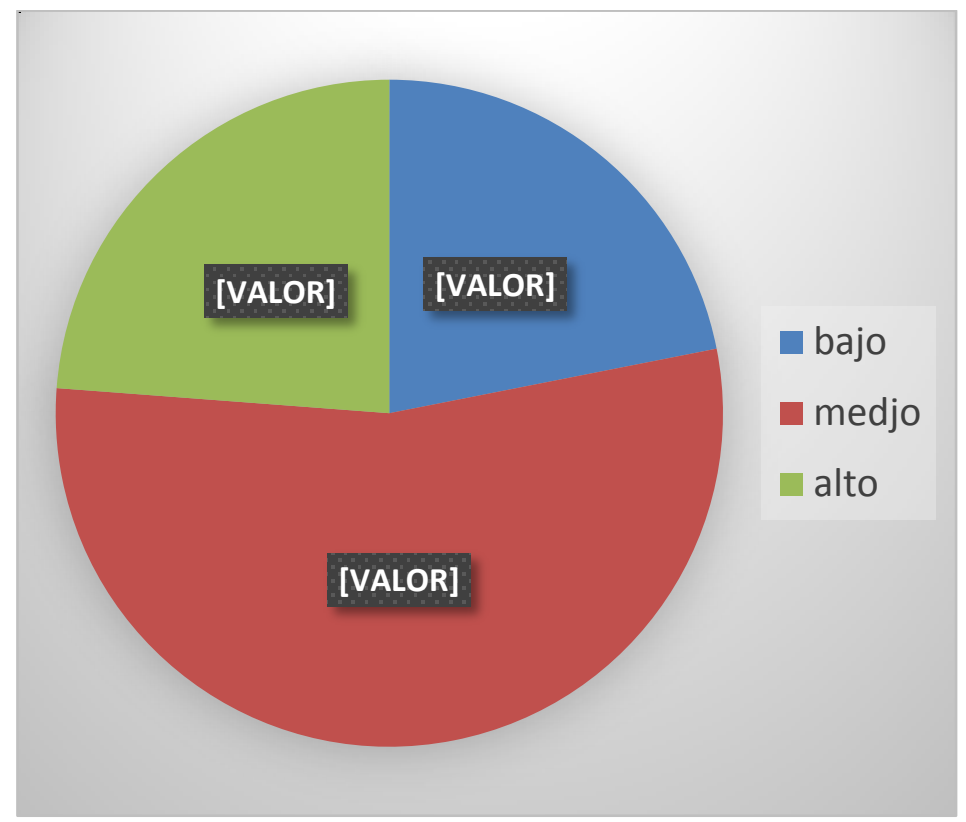

Gráfico 1. Resultados de Lenguaje Articulado

En el componente lenguaje expresivo (Gráfico 2) se puede observar que el 36,60\% de los participantes presentaron un nivel bajo en esta categoría del lenguaje.

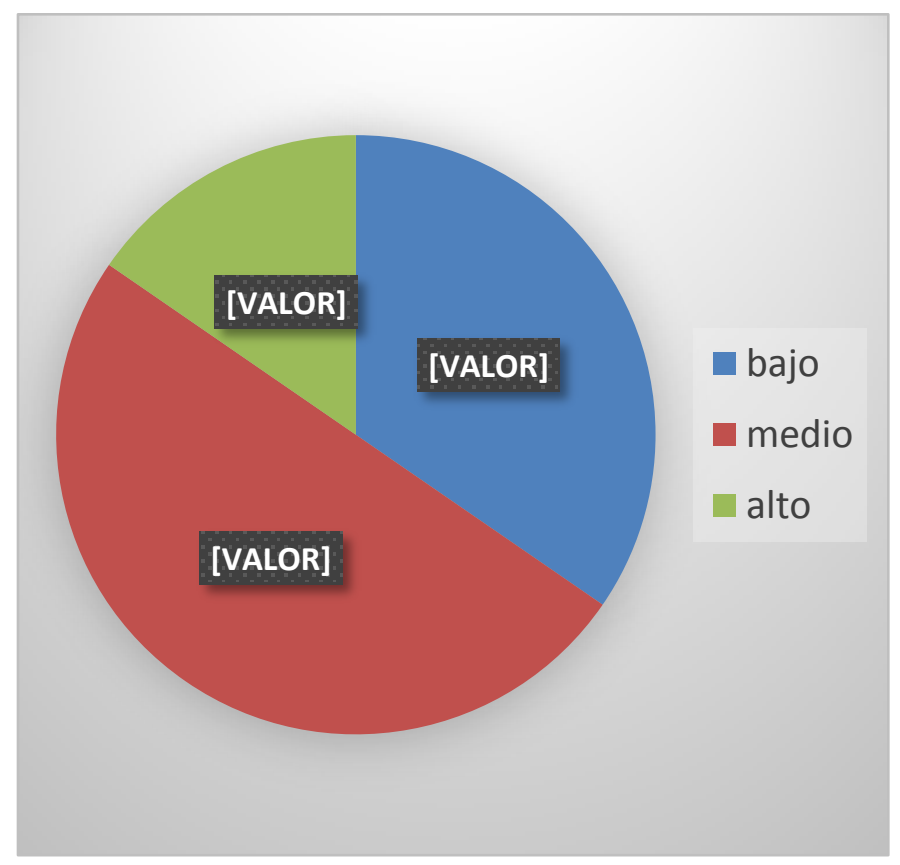

Gráfico 2: Resultados de Lenguaje Expresivo 
En la medición del componente de Lenguaje Comprensivo se observa que el 42,70\% de los participantes presenta un nivel de madurez por debajo de su edad cronológica.

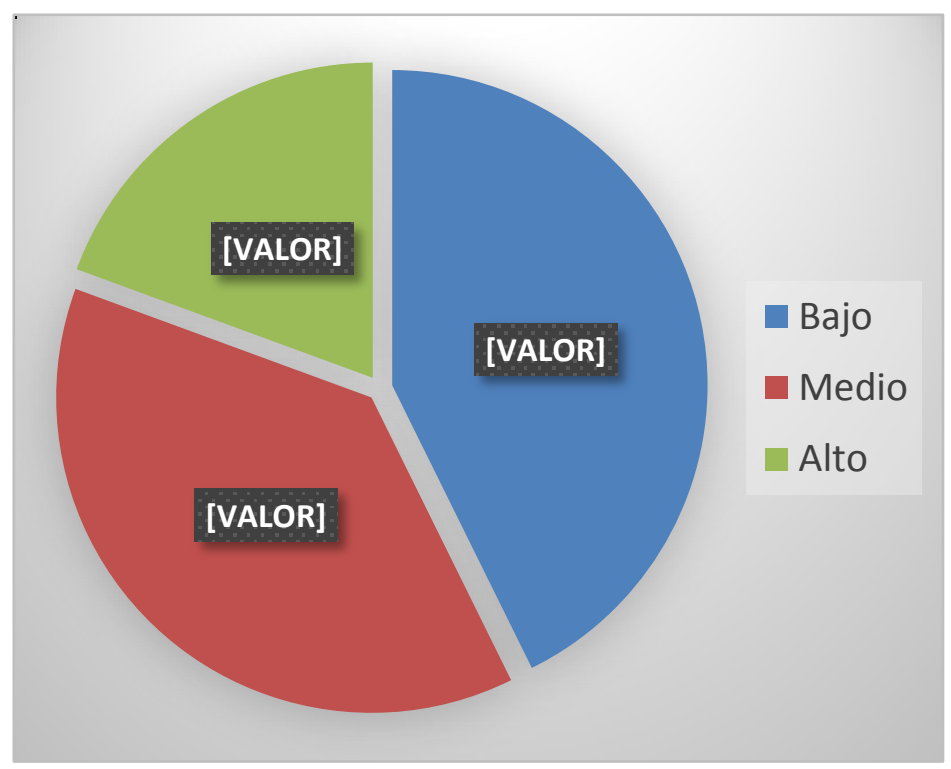

Gráfico 3. Resultados de Lenguaje Comprensivo

Para el análisis de comunicación se tomó en cuenta la categoría de Desarrollo verbal como componente importante del Lenguaje. En este componente los resultados indican que el 68,60\% de los participantes se encuentran en un nivel madurativo acorde a su edad. (Gráfico 4).

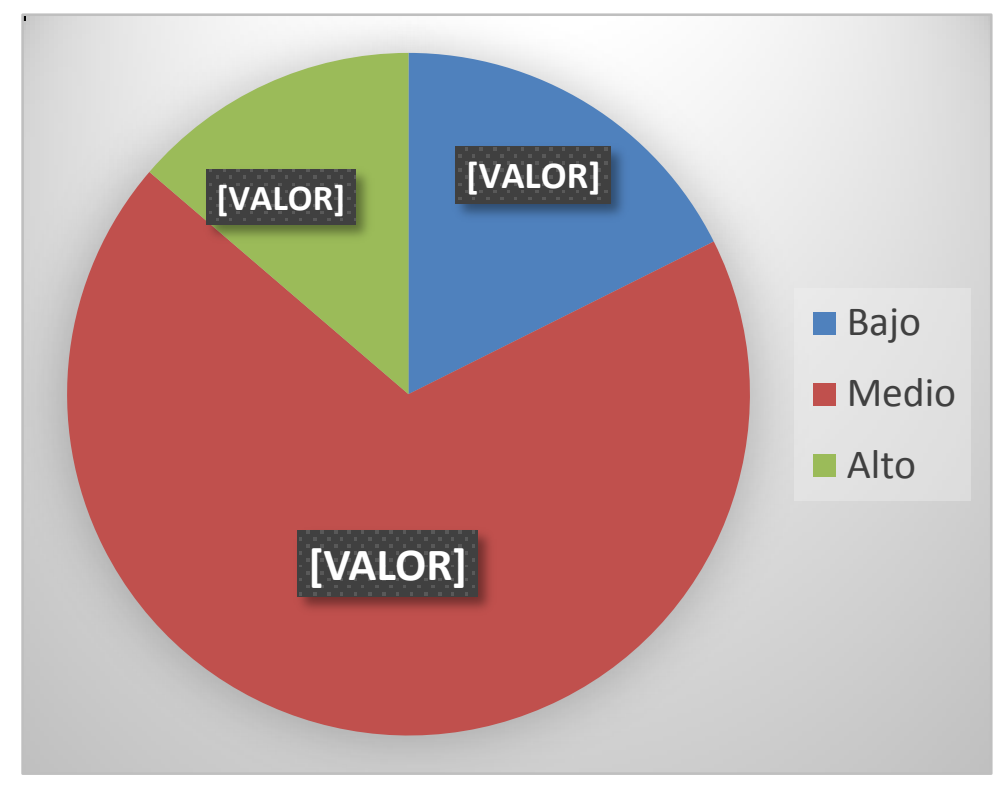

Gráfico 4. Resultados de Lenguaje Comprensivo 
En el análisis correlacional de las variables (tabla 1) se puede observar la importancia del lenguaje articulado como base para el desarrollo de las categorías de Expresión y Comprensión del Lenguaje. Además, es la principal categoría para el desarrollo verbal, entre más nivel madurativo de lenguaje articulado mejor desarrollo verbal va a caracterizar al infante $(r=0,874$, $\mathrm{p}=0,000)$.

A la vez se puede observar la escalera madurativa en cada uno de los componentes, cada uno precede a otro, de allí la importancia de poder atender a los períodos sensibles del lenguaje en la madurez de cada una de las categorías.

Tabla 1. Correlación entre categorías del Lenguaje

\begin{tabular}{|c|c|c|c|c|c|}
\hline & & $\begin{array}{l}\text { Lenguaje } \\
\text { Articulado }\end{array}$ & $\begin{array}{l}\text { Lenguaje } \\
\text { Expresivo }\end{array}$ & $\begin{array}{c}\text { Lenguaje } \\
\text { Comprensivo }\end{array}$ & $\begin{array}{c}\text { Desarrollo } \\
\text { Verbal }\end{array}$ \\
\hline \multirow[t]{2}{*}{ Lenguaje Articulado } & $r$ & 1 & & & \\
\hline & $p$ & & & & \\
\hline \multirow[t]{2}{*}{ Lenguaje Expresivo } & $r$ & 0,389 & 1 & & \\
\hline & $p$ & 0,000 & & & \\
\hline \multirow[t]{2}{*}{$\begin{array}{l}\text { Lenguaje } \\
\text { Comprensivo }\end{array}$} & $r$ & 0,16 & 0,405 & 1 & \\
\hline & $p$ & 0,107 & 0,000 & & \\
\hline \multirow[t]{2}{*}{ Desarrollo Verbal } & $r$ & 0,874 & 0,66 & 0,573 & 1 \\
\hline & $p$ & 0,000 & 0,000 & 0,000 & 0,000 \\
\hline
\end{tabular}

\section{Discusión}

El desarrollo del lenguaje, en base a las pruebas aplicadas a los 200 niños y niñas de entre 4 y 6 años de edad, está marcado como medio, aspecto que puede considerarse como normal, sin embargo, se dan a conocer un pequeño pero preocupante porcentaje de niños cuyo nivel de adquisición de características lingüísticas se encuentran por debajo del promedio. De forma que se está evidenciando un leve pero incuestionable retraso en cuando la adquisición del lenguaje de los mismos, sobre todo porque se evidencia que en las 3 subpruebas de la prueba del CUMANIN que tienen que ver con el lenguaje (articulado, expresivo y comprensivo), se muestra resultados iguales.

Los infantes evaluados por la autora Bonilla (2016), expresan un promedio de 3,82 de un máximo de 5 puntos en la evaluación de los aspectos que tienen que ver con el contenido de las palabras y el lenguaje a nivel expresivo y expresivo, que si bien hace referencia a más de la mitad, también se evidencia que existe un retraso en alguno de ellos que está presenta, lo que da relación a lo que se está estudiando en esta investigación.

Según Mendieta (2000), el cual evaluó a un total de 541 niños y niñas, expresa que los resultados del subtests Comprensión de textos aplicados a su muestra, evidencia un desempeño bajo debido a que los porcentajes de resultados obtenidos que fluctúan entre $23 \%$ a $41 \%$.

De acuerdo con el estudio (Segura, et al., 2013) en Comprensión Auditiva, el 63.3\% de sus evaluados mantuvieron resultados arraigados a un rango normal, en Expresión Comunicativa, se evidenciaron casi los mismos resultados que fluctuaban entre $66.7 \%$ y el $60 \%$, sin embargo, comenta que el $3.3 \%$ de los evaluados obtuvieron una 
calificación tan baja que debe estudiárselos como casos con retraso en dichas características. De manera que se puede evidenciar la necesidad de una estimulación para aquellos infantes cuyo desarrollo, a pesar de este ser natural, se está viendo afectado de alguna manera.

Se puede entender, por lo tanto, que el bienestar infantil se ve directamente afectado cuando se evidencia retrasos en el desarrollo de procesos que poseen la característica de ser normal y automático, debido a que o se están dando de forma incorrecta o con retrasos porque el infante está pasando por situaciones que lo afectan significativamente o a su vez, este retraso ha desencadenado una serie de situaciones que están afectado directamente al menor. De cualquier manera, es imprescindible que se busque la forma de evitar el surgimiento de factores que perturben su crecimiento tanto físico, como psicológico y social.

En base al desarrollo del lenguaje de forma general, (Blumenfeld, et al., 2018) expresa que del total de participantes de su estudio (138), 16 evidenciaban un retraso en este proceso, los cuales poseían una prevalencia de 5 veces mayor si se evidenciaba algún antecedente familiar con este aspecto y 4 veces más, si se trata de un comportamiento que se categoriza como problemático. El estrés pre natal, también se posiciono como una de las posibles causas del retraso, aunque su incidencia es menor, no se descarta la posibilidad.

\section{CONCLUSIONES}

Los participantes que han recibido espacios de estimulación temprana presentan mejores niveles de madurez en el lenguaje. Esto se debe a que a dichos infantes lo han inmiscuido en espacios en los que se destinaban, de forma única, el desarrollo y la adquisición del lenguaje, de manera que a lo largo de su crecimiento y más aún inmerso dentro de este medio, dicho proceso era mucho más factible y fácil, en base a la ayuda y previo conocimiento que recibe en estos entornos, así como la preparación del niño para que dicho aspecto se dé.

Sin embargo esto no quiere decir que quienes no desarrollan una estimulación temprana del lenguaje, no puedan adquirirlo, ya que este se debe dar de manera normal, a pesar de ello, los niños y niñas en etapas de adquisición del lenguaje, pueden presentar retrasos que dificulta su desenvolvimiento en el entorno que los rodea, debido a que los componente como la comprensión y la expresión están limitados o nulos en base a la ausencia del lenguaje y su desarrollo de forma normal en las edades promedios normales.

De esta manera se puede determinar que los principales predictores del lenguaje son el lenguaje comprensivo y expresivo, ya que le permite, como sus palabra mismo lo indica, comprender y expresar las ideas que su entorno y su propio ser desarrollan, de forma que puedan comunicarse de forma adecuada y efectiva, en esto se basa el desarrollo del lenguaje y la comunicación y de esto depende muchas etapas posteriores que tienen que ver con el lenguaje, como la escritura, lectura, etc.

Es por ello que el desarrollo del lenguaje influye en el bienestar infantil y más aún si el niño o niña no ha desarrollado dichos componentes comprensivos y expresivos, lo que hace que su desenvolvimiento en su entorno o sociedad sea limitado o nulo, produciendo afectaciones directas en su bienestar. El correcto desarrollo del leguaje y de la comunicación permite al niño conectarse con el medio que lo rodea y aprender lo necesario de el para el progreso y crecimiento personal en todos los elementos que rigen al ser humano (biopsicosocial). 
Una limitación del estudio fue no contar con el mismo número de participantes por grupo de edad, sobre todo porque existe un tabú en cuanto a la adquisición del lenguaje, ya que se toma como un proceso que se debe desarrollar sin ayuda, por lo que, el interés de un estudio como el presente a edades tan cortas como los rangos que se pretendieron estudiar genera una desconfianza en padre, cuidadores, representantes, etc., así mismo, muchas familias prefieren esperar a que sus hijos aprendan por si solos (así sea tarde) a comunicarse y por ende desarrollar el lenguaje.

Es recomendable que los padres, tutores o representantes de infantes que se encuentren en pleno proceso de adquisición de lenguaje, puedan estimular al infante para prepararlo para dicho proceso, de modo que este no conlleve un estrés para el infante, para de esta manera asegurar bienestar infantil de los mismos.

De la misma forma, es padres, tutores o representantes, controlen lo que ven los niños en los diferentes programas de televisión, para evitar, que cierto contenido afecte su proceso de adquisición de lenguaje con la inclusión de frases sin sentido o palabras más arraigadas al extranjerismo que haga perder en el niño su identidad lingüística o de lengua.

\section{REFERENCIAS}

Albets, S. L., y De la Peña, Á. C. (2016). Lenguaje expresivo en Educación Infantil: clave para la estimulación de Inteligencias Múltiples. ReiDoCrea: Revista electrónica de investigación y docencia creativa, 5(31), 316-321. Obtenido de https://digibug. ugr.es/bitstream/handle/10481/43209/ 5-31.pdf?sequence $=1$

Billard, C. (2014). Desarrollo y trastornos del lenguaje oral en la infancia. EMC-Pediatría, 49(4), 1-11. Obtenido de https://www.sciencedirect.com/science/ article/abs/pii/S1245178914689629
Blumenfeld, A., Carrizo, O. J., D’Angelo, S. I., González, N. S., Sadras, Y., Graizer, S., . . . Salamanco, G. (2018). Retraso del desarrollo del lenguaje en niños de 24 meses en un centro de salud en la Ciudad de Buenos Aires. Archivos argentinos de pediatría, 116(4), 242-247. Obtenido de https://www.sap.org.ar/uploads/archivo s/general/files_ao_blumenfeld_46pdf_1528145127.pdf

Bonilla, S. R. (2016). El desarrollo del lenguaje oral en niños de 4 años del colegio Hans Christian Andersen. (Tesis de pregrado en Educación en Nivel Inicial) Universidad de Piura

Campo, T. L. (2009). Características del desarrollo cognitivo y del lenguaje en niños de edad preescolar. Psicogente, 341351. Obtenido de http://revistas. unisimon.edu.co/index.php/psicogente/a rticle/view/1168

Canales, R., Velarde, E., Meléndez, M., y Ligán, S. (2014). Lenguaje oral y habilidades prelectoras en niños de 4 a 6 años. Un estudio sobre marginalidad y bilingüismo en el Perú. Revista de investigación en psicología, 17(1), 107-119. Obtenido de https://revistasinvestigacion.unmsm.edu. pe/index.php/psico/article/view/8974/7 846

Castilla, P. M. (2014). Bienestar infantil: ¿es posible medirlo? Boletín médico del Hospital Infantil de México, 71(1), 61-64. Obtenido de http://www.scielo.org.mx/ scielo.php?script=sci_arttextypid=S166511462014000100010

Chávez, Z. C., Merino, Z. B., Del Mazo, M. A., Guarneros, S. A., Landero, L. P., González, E. B., y Lima, C. M. (2012). Diagnóstico del estado nutricional de los niños de uno a seis años de edad del turno completo de una estancia de desarrollo y bienestar infantil en la Ciudad de México. Revista de Especialidades Médico Quirúrgicas, 17(4), 256-260

Condo, I. R. (2019). Estrategia didáctica para desarrollar la lectura comprensiva en los estudiantes de primaria en Bolivia. Horizontes. Revista de Investigación en Ciencias de la Educación, 3(12), 267-283. 
Obtenido de https://revistahorizontes. org/index.php/revistahorizontes/article/ view/86

Díez, M. d., Pacheco, S. D., De Caso, A. M., García, J. N., y García, M. E. (2009). El Desarrollo De Los Componentes Del Lenguaje Desde Aspectos Psicolingüísticos. Revista de Psicología, 2(1), 129-135. Obtenido de https://buleria.unileon.es/handle/10612 /12192

Espinoza, P. L., Marco, T. R., y Ygual, F. A. (2017). Conciencia fonológica y resolución de problemas matemáticos en educación infantil. Revista de Logopedia, Foniatría y Audiología, 38(2), 61-68. Obtenido de https://www.sciencedirect.com/science/ article/abs/pii/S0214460317300694

González Zúñiga Godoy, C. I. (2007). Los programas de estimulación temprana desde la perspectiva del maestro. Liberabit, 13(13), 19-27. Obtenido de http://www.scielo.org.pe/pdf/liber/v13n 13/a03v13n13.pdf

Lepe, M. N., Pérez, S. C., Rojas, B. C., y Ramos, G. C. (2018). Funciones ejecutivas en niños con trastorno del lenguaje: algunos antecedentes desde la neuropsicología. Avances en Psicología Latinoamericana, 36(2), 389-403. doi:https://doi.org/ 10.12804/revistas.urosario.edu.co/apl/a. 5609

Mendieta, V. T. (2000). Niveles de desarrollo del lenguaje comprensivo en niños de segundo a sexto grado de primaria. Revista de investigación en psicología, 3(1), 115-127

Montoya, V. (2003). Lenguaje y Pensamiento. Razon y palabra(32). Obtenido de http://www.razonypalabra.org.mx/anteri ores/n32/vmontoya.htm?hc_location=ufi

Moreno, M. J., García, B. S., Godoy, M. M., y Suárez, M. Á. (2011). Intervención logopédica en un caso de negligencia infantil con graves repercusiones en el lenguaje. Boletín de AELFA, 11(2), 54-60

Paredes, T. K., y Del Rosario, Q. D. (2014). El vocabulario expresivo y comprensivo en niños varones y mujeres de seis a nueve años de edad de instituciones educativas estatales y privadas de Lima Metropolitana. (Tesis para optar el grado académico de Magíster) Pontificia Universidad Católica Del Perú. Lima, Perú. Obtenido de http://tesis.pucp.edu.pe/ repositorio/bitstream/handle/20.500.124 04/5847/PAREDES_KARIN_DELROSARIO _SOLEDAD_INSTITUCIONES_EDUCATIVAS .pdf? sequence $=1$ yisAllowed $=y$

Pedreira, M. J. (2006). Medios de comunicación y parentalidad en el desarrollo de la infancia y la adolescencia. Anales de pediatría continuada, 4(2), 145150

Ripalda, V., Macías, J., y Sánchez, M. M. (2020). Rincón de lectura, estrategia en el desarrollo del lenguaje. Horizontes Revista de Investigación en Ciencias de la Educación, 4(14), 127-138

Segura, C. A., Machado, O. S., Amaya, T. T., Alvarez, A. L., Segura, C. Á., y Doris, C. (2013). Desarrollo del lenguaje comprensivo y expresivo en niños de 12 a 36 meses. Revista CES Salud Pública, 4(2), 92-105. Obtenido de https://dialnet. unirioja.es/servlet/articulo?codigo $=4890$ 178

Vargas, L. G., Guadarrama, O. J., Rizzoli, C. A., Narcizo, C. F., Medrano, L. G., Aceves, V. D., Muñoz, H. O. (2016). Análisis y comparación curricular de las estrategias o programas para el desarrollo infantil temprano en México. Boletín médico del Hospital Infantil de México, 73(2), 90-104. Obtenido de http://www.scielo.org.mx /scielo.php?script=sci_arttextypid=S166511462016000200090

Vives, M. M. (2002). Trastornos del desarrollo del lenguaje y la comunicación. Universidad Autónoma de Barcelona, 120. Obtenido de https://d1wqtxts1xzle7. cloudfront.net/38871121/12_trastornos_ desarollo_lenguaje_comunicacion.pdf?144 3048023=yresponse-content-

disposition=inline $\% 3 \mathrm{~B}+$ filename $\% 3 \mathrm{DT}$ Tras tornos_del_desarrollo_del_lenguaje_y.pdfy Expires $=1602749435 y$ Signature $=S F w X X D$ 0rM7K1ji0GZJKctP 